\title{
Terminal end Variations and Common Pathological Abnormalities of the Basilar Artery among the Ugandan Population: a Human Autopsy Study
}

\author{
E. Nagawa ${ }^{1}$ M. Okello ${ }^{2}$ H. Kiryowa ${ }^{2}$ E. Mwaka \\ ${ }^{1}$ Department of Human Anatomy, Habib Medical School, Islamic \\ University in Uganda, Kampala, Uganda \\ 2 Department of Human Anatomy, College of Health Sciences, \\ Makerere University, Kampala, Uganda \\ J Morphol Sci 2018;35:110-115.
}

Address for correspondence E. Nagawa, BSCB, MScHA, Department of Human Anatomy, Habib Medical School, Islamic University in Uganda, P.O. Box 7689, Kampala, Uganda (e-mail: edinaga67@gmail.com).

\begin{abstract}
Keywords

- terminal end variations

- pathological abnormalities

- basilar artery

Introduction Morphological variations at the basilar artery terminal end are documented risk factors for cerebral vascular complications like atherosclerosis, thromboembolisms, and aneurysms that further predispose to fatal arterial occlusions. Pathological abnormalities of the basilar artery are usually ignored on diagnosis in third world countries despite their role in brain ischemia. There is a paucity of literature on the gross anatomy of cerebral vasculature in the Ugandan population. Therefore, this study set out to determine the terminal end variations and common pathological abnormalities of the basilar artery among the Ugandan population.

Materials and Methods This was a cross sectional study that accessed intact basilar arteries from 115 human autopsy brains (77 males and 33 females) at the Kampala City Council Authority mortuary. Arachnoid matter was removed and arteries perfused with clear water for visibility. The arteries were checked for variations including bifurcations, trifurcations, quadrifurcation, hexafuraction and pathological abnormalities. Representative photographs were taken and results presented as proportions and percentages.

Results The average age of the human cadavers was 38 years (Range 18-85 years). Normal bifurcation of the basilar artery was observed in $48.7 \%$ of the study population. Other variations included trifurcations (22.6\%); quadrifurcations (21.7\%), pentafurcations $(6.1 \%)$ and hexafurcations $(0.6 \%)$. With regard to abnormalities, 16 arteries (13.9\%) had atheromatous plaques, 21 (18.3\%) were rigid, and $26(22.6 \%)$ were tortuous. There was significant correlation between age and pathological abnormalities $(p=0.01)$

Conclusion There are several variations at the terminal end of the basilar artery in the Ugandan population. Arterial pathological abnormalities are also not uncommon and tend to increase with age. Thus, the need for routine imaging investigations in patients with cerebrovascular disorders and individuals of 40 years and above.
\end{abstract}

published online July 23,2018
DOI https://doi.org/ $10.1055 / \mathrm{s}-0038-1667193$ ISSN 2177-0298.
Copyright ( 2018 by Thieme Revinter Publicações Ltda, Rio de Janeiro, Brazil
License terms

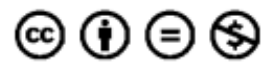




\section{Introduction}

The basilar artery formed by two vertebral arteries at the ponto medullary junction is significantly known to be the major blood source to the hind brain. It terminates into two major branches, the superior cerebellar artery and posterior cerebral arteries. It lies ventral to the pons to which it gives a series of pontine branches. Literature reviewed has shown that the basilar artery is associated with several morphological variations at its terminal end which in most cases renders it as a susceptible point for aneurysms, thromboembolisms and presenting neurosurgical complications. ${ }^{1-3}$ As a component of the vertebrobasilar circulation it supplies the pons, the cerebellum and the midbrain thus its occlusion can highly cause infarctions in these areas. Occlusion of the basilar artery is reported to contribute $8-14 \%$ of posterior circulatory stroke. ${ }^{4}$ The basilar artery is also an important factor in the pathophysiology of vertebrobasilar insufficiency that is characterized by transient ischaemia to the vertebrobasilar circulation. ${ }^{5}$ About $20 \%$ of cerebrovascular accidents involve the posterior cerebral circulation. ${ }^{6}$ There is dearth of literature on stroke from sub-Sahara Africa ${ }^{7}$ but recent figures show that it has an annual incidence of 316 per 100,000 population. ${ }^{8}$ To improve on the diagnosis of posterior vascular occlusion incidents, it is paramount that specific anatomical/morphological variations in vertebrobasilar circulation are explored.

Several morphological studies have documented the terminal end variations of the basilar artery among various populations including bifurcations, trifurcations, quadrifurcations and pentafurcations. ${ }^{9-12}$ These multiple branching patterns are believed to be lodging points for thromboembolic phenomena and pathologies like atherosclerosis and aneurysms. Aneurysms with multiple branching patterns at such points would lead to nerve compressions resulting into optical abnormalities. ${ }^{13}$

Most studies on pathologies of the basilar artery have used radiological imagery and just a few have used cadaveric specimens. ${ }^{11,14,15}$ Thus, this cadaveric study set out to describe morphological variations at the terminal end of the basilar artery and also document the common macroscopic pathologies affecting this artery.

\section{Materials and Methods}

A hundred and fifteen human cadaveric brains with intact basilar arteries and terminal branches were acquired on autopsy from the Kampala City Council Authority mortuary, Uganda for this cross-sectional study. Most of the brains were obtained from victims of road traffic accidents, mob justice and a few abandoned patients from the adjacent national referral hospital ( - Table $\mathbf{1}$ ).

A transverse incision was made across the forehead connecting the mid-ear points bilaterally. A coronal incision was then made joining the two mid-ear points. The scalp was reflected from the incised points to expose the skull. The skull was then opened manually using a metallic saw to remove the calvarium and expose the dura mater. With the supervision of a pathologist, mortuary attendants carefully removed the brains from the cranium, after sectioning just inferior to the medulla oblongata to avoid severing the arteries under study. All brains with damaged arteries were excluded from study (-Fig. 1). The specimens were then washed under running water and placed on a study tray. The base of the brains was exposed and the arachnoid mater carefully removed to avoid rapture. One end of the vertebral artery was tied with a soft string and the other perfused with clear water using a hypodermic syringe to engorge the arteries and improve on the visibility of the terminal basilar artery end.

Terminal end variations and pathological abnormalities of the basilar artery were observed by the first author, an anatomist and confirmed by the pathologist. Results were recorded in a laboratory book and representative photographs taken using a 14 megapixels Fuji Film digital camera. Morphologically, the number of terminal branches of the basilar artery and macroscopic pathological abnormalities were noted. Pathological abnormalities were defined as, the presence of atheroma plaques plus their locations along the basilar artery, rigidity, tortuosity and aneurysms. Data was analyzed using SPSS version 23 and summarized using descriptive and inferential statistics. The relationship between age pathological abnormalities was analyzed using Pearson's Chis squared test. A P-value of $<0.05$ was considered statistically significant.

Table 1 Demographic characteristics

\begin{tabular}{|c|c|c|c|c|c|c|}
\hline \multicolumn{7}{|c|}{ Study population -115} \\
\hline \multirow[t]{2}{*}{ Sex } & \multicolumn{2}{|l|}{ Age } & \multicolumn{2}{|l|}{ Height } & \multicolumn{2}{|l|}{ Cause of death } \\
\hline & Range & Population & Range & Population & Cause & Population \\
\hline Males- 77 & $0-24$ & 20 & $<150$ & 29 & Road traffic accidents & 60 \\
\hline Females- 33 & $25-29$ & 31 & & & Mob justice & 20 \\
\hline & $40-54$ & 15 & $151-175$ & 56 & Abandoned patients & 30 \\
\hline & $\geq 55$ & 19 & & & Unknown & 17 \\
\hline & Unknown & 30 & $>175$ & 30 & & \\
\hline Total & & 115 & & 115 & & 115 \\
\hline
\end{tabular}




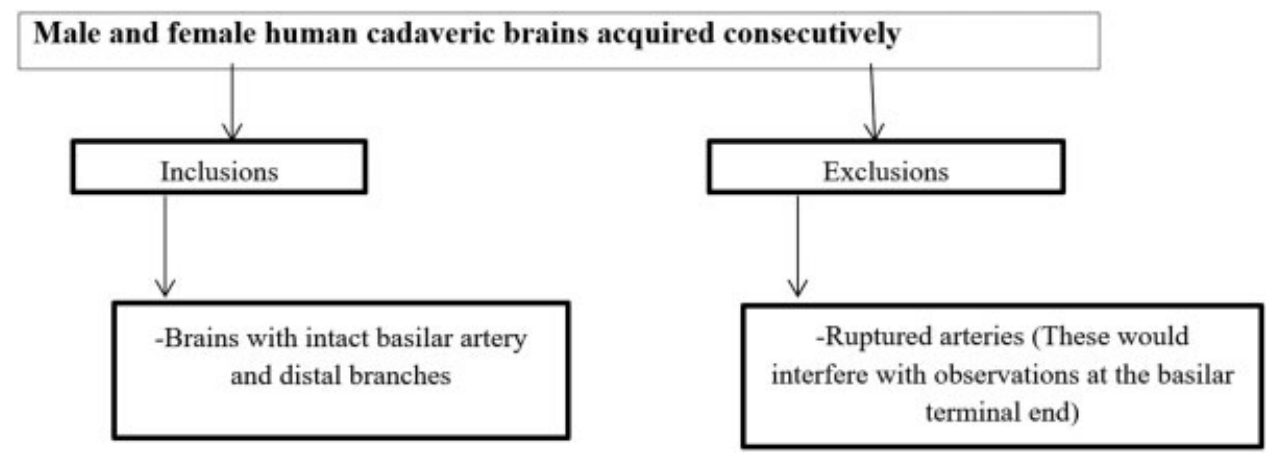

Fig. 1 Selection criteria for the study population.

The ages of victims of mob justice and traffic accidents who did not have identification records on them at the time of death could not be documented.

This study was approved by the Makerere University School of Biomedical Sciences Higher degrees and Research Ethics Committee (SBSHDREC) (Ref. No: SBSHREC-443). Administrative clearance to access cadaveric specimens at the Kampala City Council Authority (KCCA) mortuary was obtained from the mortuary In-charge and the Head of Forensic Pathology at Uganda Police Headquarters. A waiver of consent was obtained from SBSHDREC. All cadavers and specimens were treated with utmost respect and no personal identifiers were recorded to maintain confidentiality.

\section{Results}

One hundred fifteen cadaveric brains were included in this study, of which 38 (33\%) were female and 77 (67\%) male. The mean age was 38 years ( $\mathrm{SD} \pm 19$, range $18-85$ years). The majority of specimens $(56 / 115,48.7 \%)$ had normal bifurcation while trifurcations, quadrifurcation, pentafurcation and hexafurcation were also observed as summarized in - Table 2. One unique case of hexafurcation was observed with triplicated right and duplicated left Superior superior cerebellar arteries arising bilaterally from the terminal end

Table 2 Terminal end variations of the basilar artery among the Ugandan population*

\begin{tabular}{|l|l|l|}
\hline Terminal end variations & Frequency & Percent \\
\hline Bifurcation & 56 & 48.7 \\
\hline Hexafurcation & 1 & 0.9 \\
\hline Pentafurcation & 7 & 6.1 \\
\hline Quadrifurcation & 25 & 21.7 \\
\hline Trifurcation & 26 & 22.6 \\
\hline Total & 115 & 100.0 \\
\hline
\end{tabular}

${ }^{*}$ Termination variations.

Bifurcation - termination into 2 Posterior cerebral arteries branches; Triplication - termination into 3 branches 2PCAs and 1 Superior cerebellar artery; Quadrifurcation - termination into 4 branches 2SCAs and 2 PCAs at the same point; Pentafurcation - termination into 5 branches, 2 duplicated SCA, 1 single SCA, and 2 PCA; Hexafurcation - termination into 6 branches, 3 triplicated SCAs, 2 duplicated SCAs, 2 PCAs.
(-Fig. 2). There were no differences in morphology between both sexes $(p=0.55)$.

Sixteen specimens (14\%) had pathological lesions (atheroma plaques) of the basilar artery; with $14 / 16$ being 50 years of age and above. The most common pathological abnormality was rigidity (22.6\%); other abnormalities included tortuosity (-Fig. 3) and there were no aneurysms seen (-Table 3). We also investigated the location of the

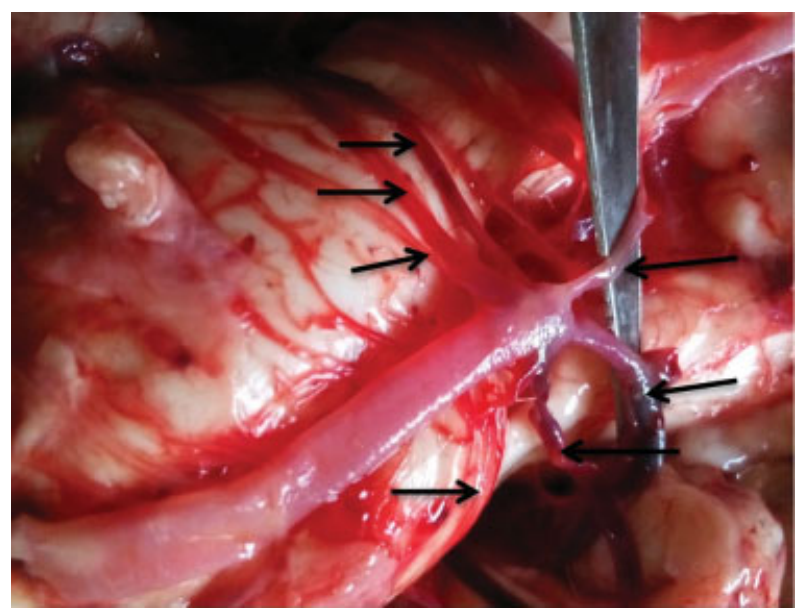

Fig. 2 Hexafurcation at the terminal end of the Basilar artery. Arrows point to the terminal branches at the distal end of the basilar artery. Note the right triplication and the left duplication of the superior cerebellar arteries.

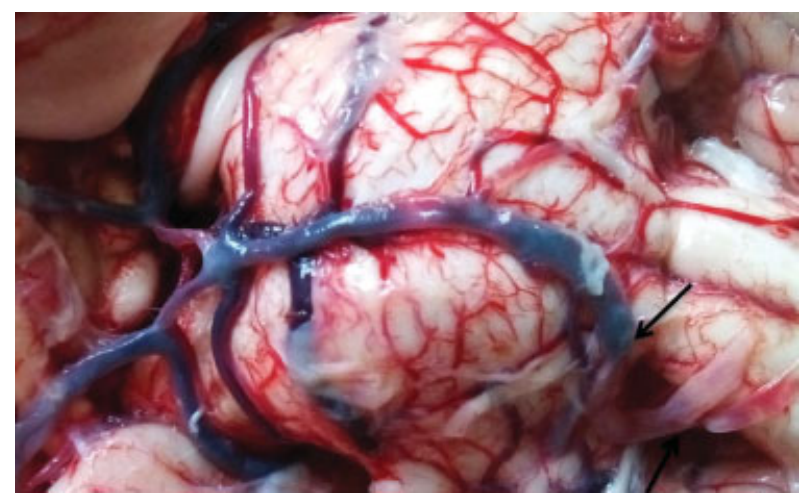

Fig. 3 Tortuosity of the basilar artery. Arrows point to the tortuous proximal end of the basilar artery. Blood supply is cut off from the two vertebral arteries. Note: Quadrifurcation at the terminal end. 
Table 3 Pathological vascular abnormalities

\begin{tabular}{|l|l|l|l|l|l|l|l|l|}
\hline \multicolumn{2}{|l|}{ Pathological abnormality } & \multicolumn{1}{l|}{ Rigidity } & \multicolumn{3}{l|}{ Tortuosity } & \multicolumn{4}{l|}{ Aneurysm } \\
\hline & Atheroma & $(\%)$ & Cases & $(\%)$ & Cases & $(\%)$ & Cases & $(\%)$ \\
\hline & Cases & 13.9 & 26 & 22.6 & 21 & 18.3 & 0 & 0 \\
\hline Presence & 16 & 86.1 & 89 & 77.4 & 94 & 81.7 & 115 & 100 \\
\hline Absence & 99 & 100 & 115 & 100 & 115 & 100 & 115 & 100 \\
\hline Total & 115 & & & & & & \\
\hline
\end{tabular}

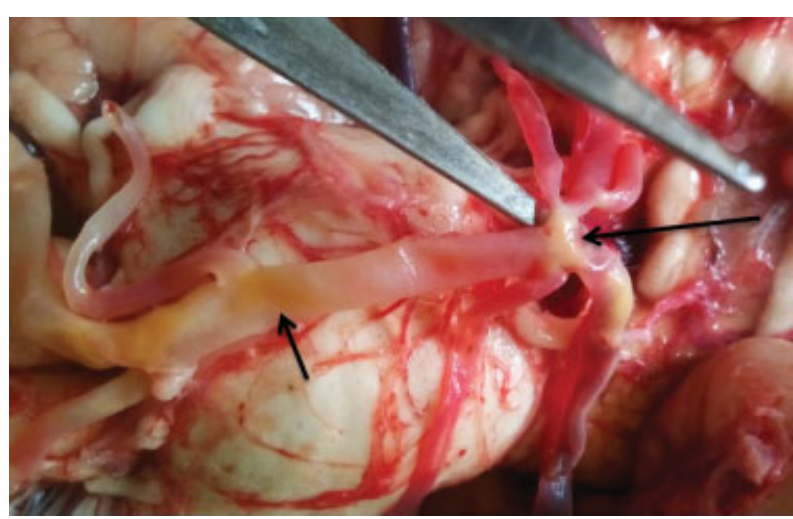

Fig. 4 Atherosclerosis of the basilar artery. Arrows point to parts with atheromatous plaques. Note: the terminal end trifurcates and the right superior cerebellar artery shares a common trunk with the posterior cerebral artery (longer arrow).

Table 4 Location of atheroma plaques along the basilar artery*

\begin{tabular}{|l|l|l|}
\hline Location point & Frequency & Percent \\
\hline Formation point & 2 & 1.7 \\
\hline Termination point & 3 & 2.6 \\
\hline Entire artery & 10 & 8.7 \\
\hline Formation and termination points & 1 & 0.9 \\
\hline No atheroma plaques & 99 & 86.1 \\
\hline Total & 115 & 100.0 \\
\hline
\end{tabular}

*Atheroma plaques along the entire artery were observed among elderly individuals above 50 years.

pathological lesions where $10 / 16(62.5 \%)$ of atheroma plaques were located along the entire length of the basilar artery (-Fig. 4). Other plaques were located either at the formation point or termination or both points of the basilar artery $(6 / 16,37.5 \%)$ (-Table 4 ). There was strong correlation between age and pathological abnormalities $(p=0.001)$.

\section{Discussion}

This study investigated the morphological variations of the terminal end of the basilar artery and also documented the common macroscopic pathologies affecting this artery in a selected Ugandan population. Almost half of the study population had normal bifurcation but there were several other variations in the branching patterns. We also found several age-related pathological abnormalities of the basilar artery. Knowledge on the terminal end variations of the basilar artery is essential when investigating arterial occlusion in the posterior circulatory system. This is essential because the vertebrobasillar circulation supplies the entire hind brain and a portion of the midbrain; therefore, lesions of the basilar artery may culminate in fatal complications considering the extent of its supply.

We report a higher percentage of basilar artery terminal end variants from the normal bifurcation with more than half of the study population (51.3\%) being either trifurcations, quadrifurcations, pentafurcations or hexafurcations. Similar studies have reported the same variations though with a varying prevalence rates. ${ }^{10,11}$ Ogeng'o et al. ${ }^{11}$ reported $82.1 \%$ normal bifurcation in a black Kenyan population and Gunnal et al. $^{10} 82.5 \%$ in a turkish population. These two studies reported a significantly higher prevalence of bifurcations as compared with our study. The most common variant terminations were trifurcations (22\%) and quadrifurcations (23\%). Similar terminal variants were reported by Ogeng'o et al. ${ }^{11}$ but with a lower prevalence. Uganda is bordered by Kenya to the East and both countries have black populations however there are notable differences in the occurrence of morphological variants. One limitation of this and Ogeng'o et al. ${ }^{11}$ studies is the small sample size. This therefore calls for a wider in-depth investigation with larger numbers.

The difference in frequencies of these variations among different populations is unknown however the similarity in morphological nature can only be explained embryologically. For example, Bala et al. ${ }^{9}$ theorizes that these variations particularly trifurcation result from lack of normal fusion of the basilar artery at the origin of the superior cerebellar artery during embryogenesis. Aydin et al. ${ }^{16}$ proposed the mal-fusion of the basilar artery at the origin of the superior cerebellar artery during embryogenesis of the basilar artery from the primitive neural arteries. They further theorize that the posterior cerebral artery anastomoses with the basilar artery caudally at a point lower than the normal site. This abnormal fusion, leads to multiple branches bifurcating close to the trunk of the basilar artery instead of the normal lateral bifurcation, with the number of terminal branches depending on the extent of fusion. ${ }^{17}$ These multiple branching points interrupt the haemodynamics resulting into aneurysms and thrombo-embolism.

In one unique case of hexarfurcation, the basilar artery terminated into 5 SCAs and 2 PCAs (-Fig. 1). Ogeng'o et al., ${ }^{18}$ 
reported a similar case of hexafurcation though with prominent therlamoperforator arteries. The increase in number of terminal branches is most likely to decrease the angles of bifurcation of the basilar artery which in turn will increase the rate of arterial occlusion. ${ }^{19}$ The high prevalence of arterial variants could predispose to age-related pathological abnormalities such as aneurysms and thromboembolic phenomena in cerebral circulation. ${ }^{20}$

The most frequent pathological abnormalities observed macroscopically were rigidity. These were observed in $22.6 \%$ most of which were associated with atheroma plaques. The cause of cerebral arterial rigidity is associated with cardiovascular and renal malfunction, since these are the predominant blood pressure regulators. Multiple branching patterns of the terminal end of the basilar artery tend to alter the hemodynamics resulting in arterial remodeling secondary to hypertension; this in turn increases arterial rigidity, plaque depositions and atherosclerosis limiting brain perfusion. ${ }^{21}$ Atheroma plaques were observed in 16 cases (14\%) within the age range of 38-85 years from the current study. Those formed at the formation and termination ends were frequent among age groups of 3850 and those that spread entirely across the arteries were among individuals with 50 and above years This could imply that atheroma plaques are most likely to commence at the basilar termination and formation points but spread as the person ages. In comparison to another study by Songur et al. ${ }^{22}$ atheromatous plaques are most likely to be frequent among individual of 50 years and above. Terminal end variations of the basilar artery, with increase in age are relatable factors to formation of atheromatous plaques.

Tortuosity cases are usually detected on MRI though these are missed out this being a non-common procedure among the third world countries. We noticed 1 severe case of tortuosity (with the rest being mild cases) which could have compromised blood supply or caused accumulation of blood in the basilar artery (-Fig. $\mathbf{3}$ ). It was noted that the basilar artery in this case was extremely longer and highly folded at the ponto medullary junction. Severe cases of tortuosity are usually associated with fatal effects of ischemic attacks. ${ }^{23,24}$ The obvious cause of tortuosity is unknown but, atherosclerosis, and aging are culminating the risk factors. ${ }^{25}$

Tortuosity like atheroma and rigidity were common cases among individuals above 40 years from our study hence age can be considered as a determining risk factor for occurrence of pathological vascular abnormalities of the basilar artery.

\section{Conclusion}

There are several morphological terminal end variants of the basilar artery among the Ugandan population. Arterial pathological abnormalities are also not uncommon and tend to increase with age. Thus, the need for routine imaging investigations in patients with cerebrovascular disorders and individuals of 40 years and above. Generalization of our findings may not be practical due to the limited scope of the study and small sample size.

\section{Acknowledgments}

This study was conducted with the assistance of pathologists; Dr. Byaruhanga Moses, Dr. Male Mutumba, Dr. Kalungi Sam and mortuary attendants of the KCCA mortuary.

\section{References}

1 Dagcinar A, Kaya AH, Aydin ME, et al. The superior cerebellar artery: anatomic study with review. Neurosurg Q 2007;17(03): 235-240

2 Ingebrigtsen T, Morgan MK, Faulder K, Ingebrigtsen L, Sparr T, Schirmer $\mathrm{H}$. Bifurcation geometry and the presence of cerebral artery aneurysms. J Neurosurg 2004;101(01):108-113

3 Wankhede HA, Hosmani P, Nimje DA. Morphological study of the basilar artery in adult human cadavers. Int J Anat Res 2014;2(03): 497-502

4 Becker KJ. Vertebrobasilar ischemia. New Horiz 1997;5(04): 305-315

5 Lima Neto AC, Bittar R, Gattas GS, et al. Pathophysiology and diagnosis of vertebrobasilar insufficiency: a review of the literature. Int Arch Otorhinolaryngol 2017;21(03):302-307

6 Moubayed SP, Saliba I. Vertebrobasilar insufficiency presenting as isolated positional vertigo or dizziness: a double-blind retrospective cohort study. Laryngoscope 2009;119(10):2071-2076

7 Mensah GA. Epidemiology of stroke and high blood pressure in Africa. Heart 2008;94(06):697-705

8 Owolabi MO, Akarolo-Anthony S, Akinyemi R, et al; Members of the H3Africa Consortium. The burden of stroke in Africa: a glance at the present and a glimpse into the future. Cardiovasc J Afr 2015; 26(02, Suppl 1):S27-S38

9 Bala M, Kaushal S, Passi DK. Trifurcation of basilar artery. Int J Anat Var 2013;6:199-200

10 Gunnal S, Farooqui M, Wabale R. Anatomical variability in the termination of the basilar artery in the human cadaveric brain. Turk Neurosurg 2015;25(04):586-594

11 Ogeng'o J, Olabu B, Obimbo M, Sinkeet S, Inyimili M. Variant termination of basilar artery in a black Kenyan population. J Morphol Sci 2012;29:91-93

12 Padmavathi G, Rajeshwari T, Niranjana Murthy K. Study of the variations in the origin \& termination of basilar artery. Anat Karnataka 2011;5(02):54-59

13 Uchino A, Sawada A, Takase Y, Kudo S. Variations of the superior cerebellar artery: MR angiographic demonstration. Radiat Med 2003;21(06):235-238

14 Kimura H, Takao M, Suzuki N, Kanemaru K, Mihara B, Murayama S. Pathologic study of intracranial large artery atherosclerosis in 7260 autopsy cases. J Stroke Cerebrovasc Dis 2017;26(12): 2821-2827

15 Masuda J, Tanaka K, Omae T, Ueda K, Sadoshima S. Cerebrovascular diseases and their underlying vascular lesions in Hisayama, Japan-a pathological study of autopsy cases. Stroke $1983 ; 14(06)$ : 934-940

16 Aydın ME, Kaya AH, Kopuz C, Demir MT, Çorumlu U, Dagcinar A. Bilateral origin of superior cerebellar arteries from the posterior cerebral arteries, and clues to its embryologic basis. Anat Cell Biol 2011;44(02):164-167

17 Padget DH. The development of the cranial arteries in the human embryo. Contrib Embryol 1948;32:205-261

18 Ogeng'o J, Mwachaka P, Olabu B, Ogeng'o N, Gimongo J. Hexafurcation of basilar artery in a black kenyan population. Austin J Anat 2016;3(01):1046

19 Stapleton CJ, Kumar JI, Walcott BP, et al. The effect of basilar artery bifurcation angle on rates of initial occlusion, recanalization, and retreatment of basilar artery apex aneurysms following coil embolization. Interv Neuroradiol 2016;22(04):389-395 
20 Karim FA, Talukdar KL, Sarma J. A study on the anatomical pattern of the anterior communicating artery in the circle of willis of the human brain with special reference to its variations in the population of assam. Indian J Res 2017;5(10):1

21 Chen Y, Shen F, Liu J, Yang GY. Arterial stiffness and stroke: de-stiffening strategy, a therapeutic target for stroke. Stroke Vasc Neurol 2017;2(02):65-72

22 Songur A, Gonul Y, Ozen OA, et al. Variations in the intracranial vertebrobasilar system. Surg Radiol Anat 2008;30(03):257-264
23 Dodevski A, Lazareska M, Tosovska-Lazarova D, Zhivadinovik J, Aliji V. Morphological characteristics of the first part of the vertebral artery. Prilozi 2011;32(01):173-188

24 Hong-tao Z, Shu-ling Z, Dao-pei Z. Two case reports of bilateral vertebral artery tortuosity and spiral twisting in vascular vertigo. BMC Neurol 2014;14(01):14

25 Ikeda K, Nakamura Y, Hirayama T, et al. Cardiovascular risk and neuroradiological profiles in asymptomatic vertebrobasilar dolichoectasia. Cerebrovasc Dis 2010;30(01):23-28 